\title{
PROPUESTA METODOLÓGICA PARA LA DELIMITACIÓN DE FAJAS MARGINALES DE LOS RÍOS
}

\author{
METHODOLOGICAL PROPOSAL FOR THE DELIMITATION \\ OF MARGINAL BELTS OF RIVERS
}

\author{
Ernesto Ramírez González
}

RECEPCIÓN: SETIEMBRE DE 2017

ACEPTACIÓN: OCTUBRE DE 2017

\begin{abstract}
RESUMEN
Un concepto clave en el estudio de la hidráulica fluvial es el establecimiento de los límites que demarcan los cauces, riberas y fajas marginales de los ríos en la actualidad. Los países de Latinoamérica carecen de herramientas necesarias para delimitar estas áreas. Una normativa general replicada en la región dirige sus esfuerzos en tratar de sustentar la teoría de las máximas avenidas como metodología de trabajo a seguir para lograr definir estos espacios. Las autoridades de agua responsables de estos trabajos se ven en la necesidad de invertir cuantiosas sumas de dinero en utilizar herramientas de ingeniería sofisticadas y software de vanguardia tratando de darle mayor sustento a su trabajo, pero los resultados finales no son satisfactorios al no ser prácticos ni aplicables en su totalidad.

La parte central del estudio es la demostración de la no aplicabilidad de la metodología hidrológica actual para delimitar el cauce, las riberas y las fajas marginales de un río. Para esto, nos ceñimos a situaciones específicas que se encuentran en el recorrido de un cauce fluvial y que indeterminan su demarcación sin dejarlas bien representadas. Finalmente, se documenta la propuesta metodológica basada en la zonificación económica ecológica y el ordenamiento territorial, la que dentro de sus puntos propone que los linderos de un cauce no deben resolverse por factores hidrológicos, sino directamente en el campo a través de conceptos hidromorfológicos y medioambientales y sociales.
\end{abstract}

Palabras clave: delimitación de fajas marginales, protección de ríos, máximas avenidas, ordenamiento territorial.

\begin{abstract}
One key element in the study of river hydraulics is to establish the limits that will draw the watercourses, banks, and belts of rivers. Currently, Latin-American countries lack the resources and tools to limit these areas; a general regulation applied through the region focuses its efforts to support the theory of maximum flood level as a work methodology to define these areas. Water authorities responsible for these works are required to invest large amounts of money and use sophisticated engineering tools and software trying to support its work, but the final result is offset since it is impractical and can't be fully applied.

The central part of the study is to demonstrate the nonapplicability of current hydraulic methodology to limit the marginal watercourses, banks, and belts of rivers, based on specific situations found in a river course which make it impossible to mark the limits. Finally, we present a methodological proposal based on ecologicaleconomical zoning and land management which proposes that the limits of a river shall not be carried out according to hydrological elements but directly on the field, using hydro morphological, environmental and social concepts.
\end{abstract}

Keywords: Delimitation of marginal belts; river protection; maximum flood; land management.

* Docente de la Escuela Profesional de Ingeniería Civil, Facultad de Ingeniería-URP <ernestoramirezimf@gmail.com> 


\section{Introducción}

La delimitación de las áreas aledañas a los ríos y las propias que definen su cauce son definiciones que deben ser establecidas por la autoridad de aguas del país. En el año 1969, se promulgó la Ley General de Aguas en el Perú, hoy Ley de Recursos Hídricos, y consecuentemente sus reglamentos e instructivos que definen o intentan definir los criterios para la delimitación del cauce, riberas y fajas marginales de los ríos, documentación que sirve como fuente única y autorizada para realización de estas delimitaciones. El presente estudio se realizó específicamente en el río Chumbao, Andahuaylas-Apurimac, como respuesta a una necesidad de la población por proteger su fuente de recurso hídrico. Este río asume la responsabilidad de abastecer de agua potable a la población y de sostener la economía a través de la irrigación y soportando el desarrollo de los distritos de San Jerónimo, Andahuaylas y Talavera.

\section{Objetivos}

- Mostrar la problemática sobre la normativa orientada a delimitar los cauces.

- Fomentar la protección de las áreas ribereńas y el ecosistema fluvial.

- Delimitar las fajas marginales del río Chumbao, en una extensión de 18 km de sección longitudinal.

\section{Metodología}

Se necesita realizar la delimitación de los linderos del lecho fluvial y de las áreas de protección del río Chumbao, ubicado en la sierra sur del Perú (departamento de Apurímac), en una extensión de 18 km de sección longitudinal que corresponde al tramo de río que atraviesa los tres distritos más importantes de la cuenca: San Jerónimo, Andahuaylas y Talavera. La delimitación busca proteger los ecosistemas fluviales de la evolución demográfica desmesurada y desordenada. Sin duda alguna, buena parte de los recursos más afectados lo constituyen los ríos y su entorno. La demanda creciente de agua se ha acrecentado en todos los sectores: rural, urbano, industrial y agrícola. Las nuevas poblaciones urbanizadas, junto con los cultivos, se asientan en los cauces, riberas y las aún inexistentes áreas de protección. Más aún, se ejecutan un sinfín de obras de acondicionamiento para que los ríos se ciñan a las necesidades de la gente.

Para efectos de la ley, los cauces son el continente de sus aguas durante sus máximas crecientes. Asimismo, las riberas constituyen las áreas de los ríos comprendidas entre el nivel mínimo de sus aguas y el que este alcance en sus mayores avenidas o crecientes ordinarias. Por su parte, las fajas marginales son bienes de dominio público hidráulico. Estas están conformadas por las áreas superiores a las riberas de las fuentes de agua naturales o artificiales y su delimitación se basa, entre otros criterios, en la máxima crecida o avenida de los ríos, sin considerar las máximas crecidas registradas por causas de eventos excepcionales. En resumen, luego de revisar los reglamentos e instructivos "explicativos" de las definiciones presentadas, se puede inferir que el lindero interior de faja marginal lo define la máxima avenida ordinaria y el exterior, la máxima avenida extraordinaria.

\section{a. Delimitación del lindero interior de faja marginal}

Con el fin de establecer los linderos respectivos, se emplearon dos métodos para el cálculo de caudales: el método probabilístico, desarrollado por la Oficina Nacional de Evaluación de Recursos Naturales 
(ONERN), y el método empírico, que toma como referencia las marcas en el terreno analizado con ayuda del software HEC-RAS.

El método de la ONERN se aplica al territorio nacional. El procedimiento consta de tres partes principales. Primero, se aplica una función de distribución teórica útil para tratar registros de valores máximos. Luego, se combinan todos los datos de una región razonablemente homogénea. Finalmente, se desarrolla una relación de máximas descargas con factores fisiográficos.

Para el cálculo de las avenidas, se utiliza la función de distribución de probabilidades de Gumbel, que se expresa de la siguiente manera:

$$
F(x)=P=e^{-e^{-y}}(1)
$$

Donde:

$$
\begin{aligned}
& \text { xi: Evento } \\
& \text { y: Variable reducida } \\
& \text { P: Probabilidad acumulativa }
\end{aligned}
$$

La variable reducida "y" está definida por las siguientes relaciones:

$$
\begin{gathered}
y=-\ln (-\ln P)(2) \\
y=\frac{\sigma_{x}}{\sigma_{x}}(x-x i)+y_{n}
\end{gathered}
$$

Donde:

$$
\begin{aligned}
& n: \text { Cantidad teórica } \\
& y_{n}: \text { Media aritmética de la cantidad teórica } \\
& x: \text { Media aritmética del evento } \\
& \sigma_{x}: \text { Desviación estandar del evento } \\
& \sigma_{n}: \text { Desviación estandar de la cantidad teórica }
\end{aligned}
$$

La ecuación se puede presentar de la forma aproximada siguiente. Si las abscisas representan el área y las ordenas la descarga máxima:

$$
q=0.5 \cdot C^{\left(1.08 \cdot A^{-4.06}\right.}(4)
$$

Donde:

$$
\begin{aligned}
& q\left(m^{3} / s\right): \text { Descarga máxima variable } \\
& A\left(K m^{2}\right): \text { Area } \\
& C(-) \text { : Coeficiente de escala adimensional }
\end{aligned}
$$

En caso se requiera considerar diferentes periodos de retorno, estas ecuaciones podrían extenderse. Existe una relación lineal, con buena aproximación, entre el logaritmo del periodo de retorno y la relación de la avenida en términos del caudal promedio. Sustituyendo el coeficiente $\mathrm{C}$ en la ecuación (4) por una relación lineal con el período de retorno como variable, se pueden considerar diferentes períodos de retorno. Después de la sustitución antes citada y la transformación de la ecuación para el cálculo de las avenidas, se obtiene otra ecuación de la siguiente forma:

$$
Q_{t}=\left(C_{1}+C_{2}\right) \cdot \log T \cdot A^{\left(1 \times-A^{-4}\right)}
$$

Donde:

$Q t(m / s)$ : avenida con periodo de retorno

$C_{1}(-)$ : coeficiente de escala adimensional

$C_{2}(-)$ : coeficiente de escala adimensional

$T(a n ̃ o):$ periodo de retorno como variable 


\section{$A\left(K m^{2}\right)$ : área como variable \\ $m(-)$ : exponente adimensional \\ $n(-)$ : exponente adimensional}

En la ecuación deducida, los exponentes m y n determinan la forma y la pendiente de la curva, y los coeficientes $\mathrm{C} 1$ y C2, la escala de la curva dependiente del período de retorno escogido. Por lo tanto, la curva se traslada de manera paralela a la ordenada subiendo para grandes períodos de retorno y bajando para pequeños períodos. Los parámetros que se requieren para la aplicación de la ecuación en las diferentes regiones se presentan en la tabla 3. Debido a que, en cuencas muy pequeñas, las avenidas tienen mucha variación y existen muy pocas observaciones, la validez de las ecuaciones se limita a cuencas cuyas áreas sean mayores de $50 \mathrm{~km}^{2}$, y menores de $30000 \mathrm{~km}^{2}$.

Las siete regiones mostradas en el mapa de regionalización de las avenidas del Perú (figura 1), han sido agrupadas en función a sus factores fisiográficos, con los cuales se determinan los coeficientes c1, c2, m y n, para cada cuenca en su respectiva región. Mientras que en la tabla 1 se presentan las regiones y las cuencas integrantes, el área aproximada, el número y la densidad de las estaciones consideradas están representadas en la tabla 2.

TABLA 1. REgIONES Y CUENCAS INTEGRANTES

\begin{tabular}{|c|l|l|}
\hline No & \multicolumn{1}{|c|}{ Región } & \multicolumn{1}{c|}{ Cuencas } \\
\hline 1 & Costa Norte & Tumbes a Piura \\
\hline 2 & Costa Norte & Cascajal a Santa \\
\hline 3 & Sierra Norte & Alto Marańón \\
\hline 4 & Costa Central & Lacramarca o Camaná-Majes \\
\hline 5 & Costa Sur & Quilca o Caplina \\
\cline { 2 - 3 } & Titicaca & Titicaca \\
\hline 6 & Sierra Central Sur & Mantaro, Apurimac y Urubamba \\
\hline 7 & Selva & $\begin{array}{l}\text { Ucayali, Bajo Marañon, Madre de } \\
\text { Dios y Amazonas. }\end{array}$ \\
\hline
\end{tabular}

TABLA 2. CARACTERÍSTICAS PRINCIPALES DE LAS REGIONES

\begin{tabular}{|c|c|c|c|c|}
\hline \multirow{3}{*}{ Región } & \multirow{2}{*}{$\begin{array}{c}\text { Área } \\
\text { A }\end{array}$} & \multirow{2}{*}{$\begin{array}{c}\text { Est. } \\
\text { N }\end{array}$} & \multicolumn{2}{|c|}{ Densidad } \\
\hline & & & $\mathrm{D}_{1}=\mathrm{A} / \mathrm{N}$ & $\mathrm{D}_{2}=\mathrm{N} / \mathrm{A}$ \\
\hline & $\left(\mathbf{K m}^{2}.\right)$ & $(-)$ & $\left(\right.$ Est $\left./ \mathrm{Km}^{2}{ }^{2}\right)$ & $\left(\mathrm{Km}^{2} /\right.$ Est. $)$ \\
\hline 1 & 31000 & 12 & 0.0004 & 2580 \\
\hline 2 & 59000 & 21 & 0.0004 & 2810 \\
\hline 3 & 64000 & 34 & 0.0005 & 1880 \\
\hline 4 & 142000 & 58 & 0.0004 & 2450 \\
\hline 5 & 101000 & 28 & 0.0003 & 3610 \\
\hline 6 & 137000 & 40 & 0.0003 & 3420 \\
\hline 7 & 751000 & 0 & -- & -- \\
\hline
\end{tabular}

Las secciones de cuenca utilizadas para el cálculo del caudal corresponden al área de influencia sobre los puntos de análisis ubicados al ingreso de los distritos San Jerónimo, Andahuaylas y Talavera. 
TABla 3. Parámetros de la ecuación (5)

\begin{tabular}{|c|c|c|c|c|}
\hline Región & $\mathbf{C}_{\mathbf{1}}$ & $\mathbf{C}_{\mathbf{2}}$ & $\mathbf{m}$ & $\mathbf{n}$ \\
\hline 1 & 1.01 & 4.37 & 1.02 & 0.04 \\
2 & 0.10 & 1.28 & 1.02 & 0.04 \\
3 & 0.27 & 1.48 & 1.02 & 0.04 \\
4 & 0.09 & 0.36 & 1.24 & 0.04 \\
5 & 0.11 & 0.26 & 1.24 & 0.04 \\
6 & 0.18 & 0.31 & 1.24 & 0.04 \\
7 & 0.22 & 0.37 & 1.24 & 0.04 \\
\hline
\end{tabular}

Para el cálculo de los caudales usando las marcas en el terreno (HEC-RAS), se realizó el levantamiento topográfico a detalle y el seccionamiento transversal del río con un espaciamiento promedio de $100 \mathrm{~m}$ entre dos secciones de corte, distancia que abarca los $18 \mathrm{~km}$ de longitud del río. Dentro de los datos hidrológicos, se analizaron los métodos y resultados obtenidos en diversos estudios realizados por la Dirección General de Aguas y Suelos (DGAS), el Proyecto de Encauzamientos de Ríos y Protección de Estructuras de Captación (PERPEC) y otros proporcionados por la Administración Técnica del Distrito de Riego. El caudal de máxima avenida ordinaria que transcurre por el río será determinado por tanteo tomando como referencia el nivel de marcas dejadas en el terreno.

\section{b. Delimitación del lindero exterior de faja marginal}

Gracias al software HEC-RAS y a la topografía del terreno, se realizó el análisis de los niveles de agua que

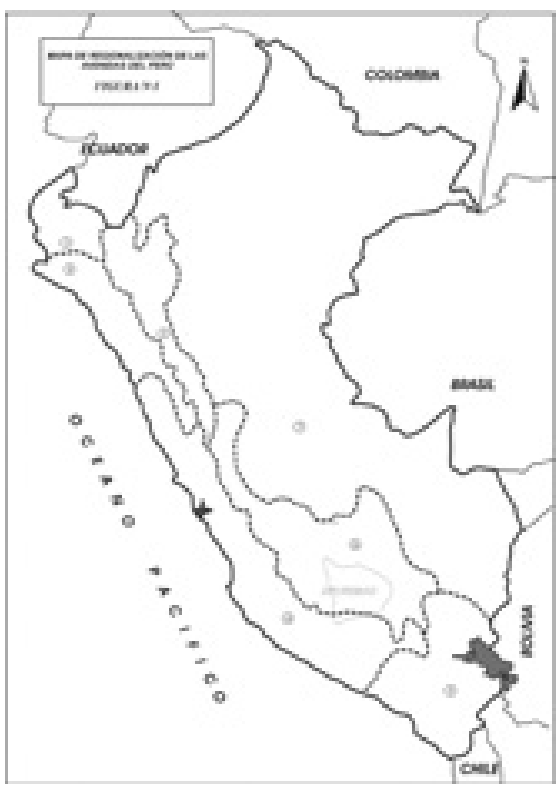

Fig. 1. Mapa de regionalización. alcanzó la avenida extraordinaria ocurrida en el año 1998. Con un caudal estimado de $140 \mathrm{~m} 3 / \mathrm{s}$, este nivel alcanzado por el agua vendría a ser el delimitante exterior.

Con estos dos linderos deberíamos tener ya definido nuestro ancho de faja en cualquier parte del río.

\section{Resultados}

La comparación de los caudales hallados por los dos métodos propuestos determina una variabilidad del cauce y lindero interior de la faja marginal.

\section{a) Cálculo del caudal método probabilístico-ONERN}

Para los distritos de San Jerónimo (Q1), Andahuaylas (Q2) y Talavera (Q3), se obtuvieron los caudales de máxima avenida ordinaria. Sustituyendo los valores en la ecuación que asocia los diferentes períodos de retorno $(\mathrm{Tr})$, resultan los valores mostrados en la Tabla 4. 
TABLA 4. CAUdALES MÉTODO PROBABILÍSTICO

\begin{tabular}{|c|c|c|c|}
\hline $\begin{array}{c}\mathrm{Tr} \\
\text { (años) }\end{array}$ & $\begin{array}{c}\mathrm{Q}_{1} \\
(\end{array}$ & $\begin{array}{c}\mathrm{Q}_{2} \\
(\end{array}$ & $\mathrm{Q}_{3}$ \\
\hline 20 & 77 & 114 & 136 \\
\hline 25 & 82 & 122 & 146 \\
\hline 50 & 100 & 149 & 177 \\
\hline 100 & 118 & 175 & 209 \\
\hline
\end{tabular}

\section{b) Cálculo del caudal método directo-HEC-RAS}

Las marcas en el terreno nos arrojaron los caudales de máxima avenida ordinaria, estimados en cada sección transversal (180 secciones), de los cuales se obtuvo un caudal representativo para cada distrito, que fueron: San Jerónimo, $40 \mathrm{~m} 3 / \mathrm{s}$; Andahuaylas $60 \mathrm{~m} 3 / \mathrm{s}$; y Talavera 80, m3/s, como se aprecia en las figuras 2, 3 y 4, que son las secciones transversales al ingreso de cada distrito respectivamente.

La diferencia de caudales fue de 37, 54 y 56 m3/s en los distritos de San Jerónimo, Andahuaylas y Talavera respectivamente. La diferencia puede ser mayor si se aumenta el periodo de retorno y el área de drenaje correspondiente al lugar de análisis. Esta diferencia de caudal significa una variación de los linderos internos de faja marginal de más de $30 \mathrm{~m}$.

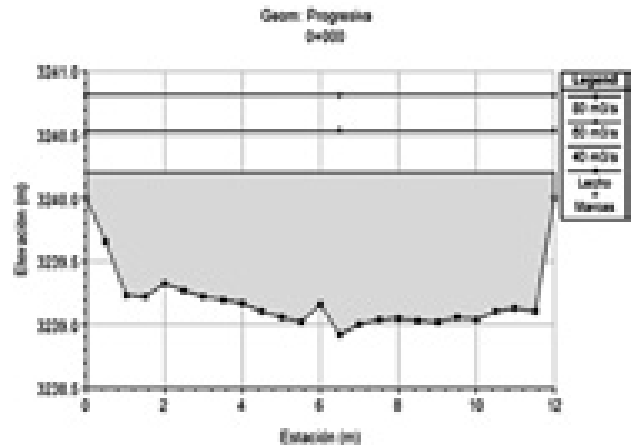

Fig. 2. Sección transversal-San Jerónimo.

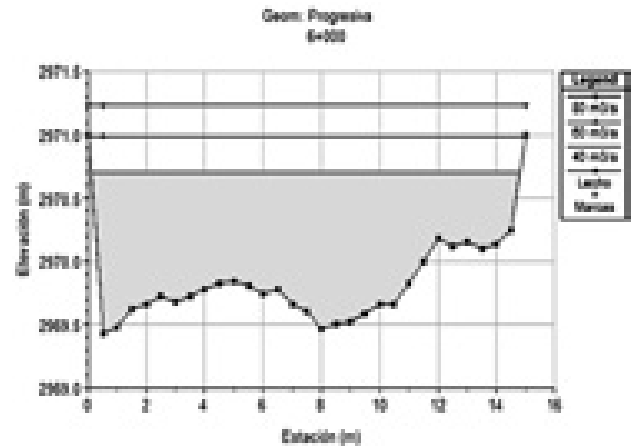

Fig. 3. Sección transversal-Andahuaylas.

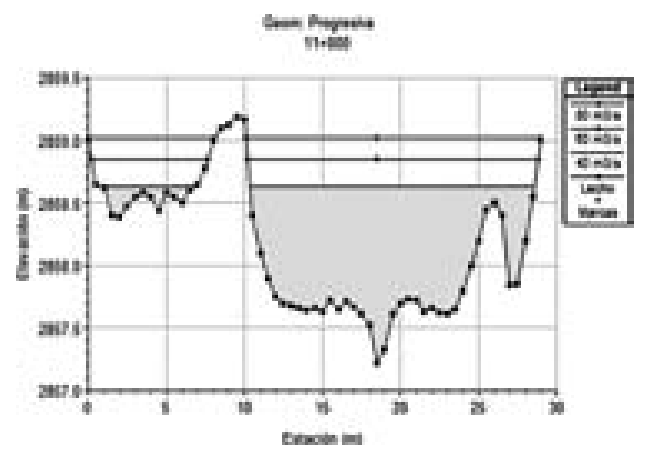

Fig. 4. Sección transversal-Talavera. 


\section{c) Análisis del lindero exterior de faja marginal por medio de las máximas avenidas extraordinarias}

Haciendo uso del programa HEC-RAS y la topografía del terreno, se realizó el análisis de zonas inundables ocasionadas por una avenida extraordinaria ocurrida en el año 1998, con un caudal estimado de $140 \mathrm{~m} 3 / \mathrm{s}$. La planicie de inundación incluyó grandes áreas de terrenos agrícolas, centros comerciales y viviendas aledañas al cauce, con mayor repercusión en el distrito de Andahuaylas.

\section{d) Propuesta metodológica para la delimitación de fajas marginales de los ríos sustentada en la zonifica- ción económica ecológica}

La propuesta está basada en los criterios aplicados en la zonificación económica ecológica y el ordenamiento territorial, con influencia directa e indirecta en el sistema fluvial, lo que nos permitirá tener las herramientas necesarias para la toma de decisiones en referencia al ancho de faja marginal. El proceso considera dos medios que deben articularse armónicamente: el físico-biológico y el socio-económico, cada uno con sus temas y características específicas.

La propuesta consta de cinco fases. La primera, llamada fase preliminar, contiene la definición de objetivos de la delimitación, el establecimiento del equipo técnico multidisciplinario y la definición del marco conceptual de referencia. La segunda, denominada fase de generación de información, comprende tareas como el análisis y evaluación de áreas aledañas al cauce, la confiabilidad de la información recopilada y la elaboración del mapa base a escala conveniente con respecto a la longitud de tramo de río a delimitar.

Dentro de la tercera fase, también llamada de análisis (ver figura 5), tenemos el medio físicobiológico con los componentes de relieve, clima, suelo, vegetación productiva y no productiva, agua superficial y subterránea, recurso energético, valor turístico, patrimonio cultural, valor ecológico y áreas protegidas por el Estado, que con relación a la escala de trabajo se caracteriza y se analiza de acuerdo a las necesidades de la delimitación misma. El medio socio-económico considera el análisis de factores como la producción de la tierra, uso actual, sistemas de producción, infraestructura económica, demografía (cultura), estructura institucional, servicios básicos y sociales, todo ello en relación a las unidades principales del medio físico-biológico, con lo cual se establece la interrelación de los dos medios.

Por su parte, en la cuarta fase, también conocida como fase de evaluación, se realiza un análisis integrado del uso de los recursos naturales y la ocupación del territorio, que permite identificar el patrón actual de uso de los recursos naturales y la ocupación del territorio, así como los problemas existentes y las potencialidades disponibles. El análisis integrado, permitirá alcanzar resultados como la descripción del patrón actual de uso y ocupación del territorio como escenario actual que se desea cambiar o fortalecer según los casos, así como la tipificación de los problemas centrales que deben ser resueltos apoyando el proceso definición de prioridades.

Finalmente, la fase de validación contempla la participación de la población involucrada. Es muy probable que surjan discrepancias y situaciones conflictivas entre la autoridad de aguas (jefe del proyecto) y la población que pueda verse afectada por la delimitación a realizar. En este sentido, se debe mostrar el interés de la autoridad por mejorar la condición social y económica de la población respetando el ecosistema fluvial. No se debe entender esto como una herramienta de coacción que puede ejercer la comunidad sobre el proyecto, sino como una forma de transparencia y equidad en el manejo de cada delimitación. 


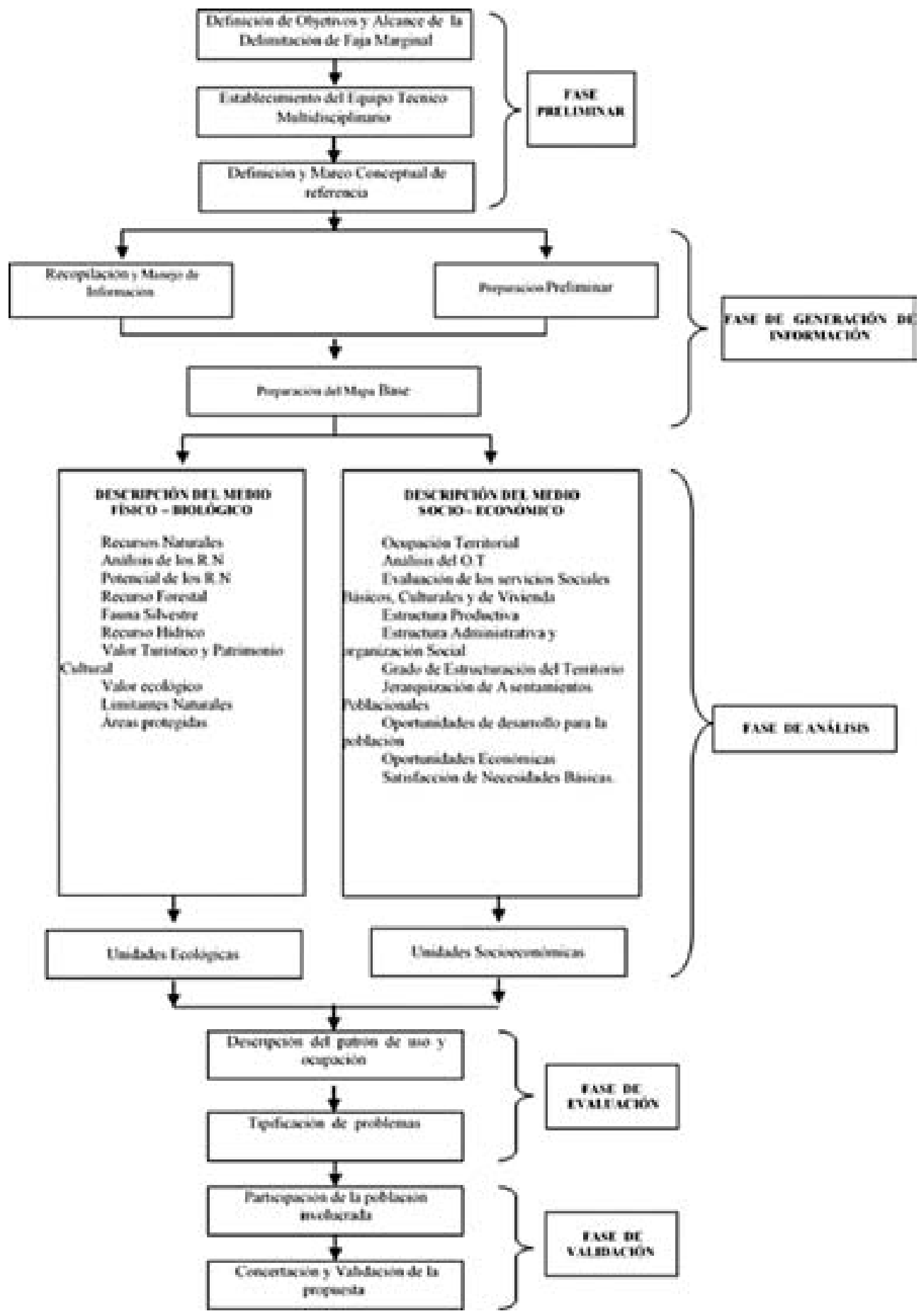

Fig. 5. Flujograma de propuesta metodológica para la delimitación de fajas marginales de los ríos, sustentado en la zonificación económica ecológica. 


\section{Discusión}

El primer punto en discusión es la ubicación de los linderos del cauce adecuado para estos proyectos. Los resultados obtenidos en los tres distritos por los métodos ya expuestos indican una marcada variabilidad. Esto significa que el cauce está en función del método hidrológico que se utilice para su cálculo.

Las márgenes de los ríos no siempre se encontraban al mismo nivel, razón por la cual, en ciertos tramos, el agua de máxima avenida extraordinaria solo se desbordó sobre una de las márgenes del cauce, lo que significa que la margen no inundada no poseería faja marginal. Esto se observó en la progresiva 9+200 km, donde la diferencia de niveles es mayor a los $3 \mathrm{~m}$.

Debido a la topografía del terreno, la vista en planta de las márgenes del río y la línea de máxima avenida extraordinaria se observaron muy irregulares y difícilmente cumplían con la equidistancia recomendada en la norma.

La máxima avenida extraordinaria inundó extensas áreas de terrenos, centros comerciales, avenidas principales, entre otros. Teniendo en cuenta que el valle en estudio es muy estrecho, se hace inviable la delimitación de dichos terrenos como áreas de fajas marginales.

La cuenca posee lagunas de cabecera que almacenan agua en épocas de avenidas para ser utilizadas en épocas de estiaje. Esta modificación de caudales hace variar el cauce del río (según la definición actual de cauce) y, por consiguiente, los linderos de faja marginal. Este hecho se observa con mayor intensidad en cuencas altamente pobladas en donde la regulación de aguas es mayor.

Los estudios de planicies de inundación se realizan con la finalidad de implementar planes de contingencia en beneficio de la población que pudiera verse afectada, mas no para delimitar áreas intangibles que se encuentran definidas por leyes y normativas específicas.

El problema con relación a las fajas marginales no son las inundaciones, sino la acelerada urbanización y la poca planificación del ordenamiento territorial en cuencas hidrográficas. Como consecuencia de los resultados obtenidos y conociendo las necesidades socioeconómicas y ambientales, se propone una metodología para la delimitación de fajas marginales de los ríos basada en la Zonificación Económica Ecológica y el Ordenamiento Territorial.

Finalmente, la ley, los reglamentos e instructivos referentes al tema presentan grandes contradicciones e indeterminaciones originadas por la complejidad del sistema fluvial. Sumado a esto, las instituciones responsables afrontan dificultades legales y de capacidad institucional para encarar temas relacionados con la protección de los ecosistemas y el medio ambiente.

\section{Conclusiones}

De la revisión de información y de los resultados obtenidos, se derivan las siguientes conclusiones:

- La posición del cauce y lindero interior de faja marginal presentan grandes variaciones según el método de cálculo (directo y probabilístico). Esto representa dos cauces diferentes para un mismo caudal fluvial.

- El criterio o metodología de zonas inundables no es adecuado para delimitar el lindero exterior de faja marginal, no solo por las dificultades presentadas en los resultados, sino también porque no introduce ningún aspecto social, ecológico, económico ni cultural necesarios para una delimitación justa.

- Existe confusión y contradicción en las diferentes normas referidas al tema, sobre todo, en cuanto a la definición de conceptos y procedimientos que llevan a diferentes interpretaciones $\mathrm{y}$, por consiguiente, a errores.

- No existe una relación causa-efecto entre las inundaciones y la delimitación de fajas marginales. 


\section{Recomendaciones}

- En general, para cada proyecto de delimitación de fajas marginales, se debe ejecutar la apertura de vías en ambas márgenes del río que permitan el libre tránsito y la vigilancia de las estructuras hidráulicas.

- Se debe unificar y estructurar mejor la normativa vigente para evitar ambigüedades y contradicciones entre ellas.

- Es necesario implementar un instructivo técnico o formato de expediente técnico que defina los trabajos a seguir por la autoridad del agua para estandarizar los procedimientos de las delimitaciones.

- Se recomienda fortalecer la capacidad de los profesionales que trabajan en procesos de planificación y desarrollo demográfico. Estos necesitan aplicar conceptos, metodologías e instrumentos para elaborar planes de ordenamiento territorial en cuencas hidrográficas.

- Se debe implementar un plan nacional de restauración de sistemas fluviales, no solo como un gran proyecto efímero, sino como una política nacional que demuestre un equilibrio entre el progreso y la naturaleza.

- Es conveniente que la responsabilidad de formular el plan propuesto recaiga sobre un equipo técnico multidisciplinario que desarrolle un análisis detallado de los diferentes factores que intervienen en el proceso de una delimitación de faja marginal.

- Es necesario implementar una nueva definición de cauce dentro de la normativa vigente que facilite su delimitación en campo.

\section{Referencias}

[1] R. Ambrosio, "Estudios de máximas avenidas y de transporte de sedimentos en el río Chancay-Huaral con fines de control de inundaciones", Tesis de grado, Facultad de Ingeniería Agrícola, Universidad Nacional Agraria La Molina, Lima, 2001.

[2] F. Meyer, "Delimitación de la faja marginal de los ríos Rímac, Lurín y Chillón", ATDR (Administración técnica del distrito de riego), Lima: ATDR Chillón-Rímac-Lurín, Informe técnico, 1, 1999.

[3] F. Meyer, "Delimitación de la faja marginal del río Santa Eulalia", ATDR (Administración técnica del distrito de riego), Lima: ATDR río Chillón-Rímac-Lurín, Informe técnico, 2, 2004.

[4] Metodología para la Zonificación Ecológica y Económica, 010-2006-CONAM, 2006.

[5] HEC-RAS River Analysis System User's Manual, 4ta. Ed., U.S. Army Center CORPS OF ENGINEERS, USA, 2010.

[6] P. Esteban, "Predicción de áreas inundables mediante la aplicación del Software HEC RAS 2.1 en un sector del río Lurín”, Tesis de grado, Facultad de Ingeniería Agrícola, Universidad Nacional Agraria La Molina, Lima, 2001.

[7] J. Gástelo, “Análisis regional de máximas avenidas con aplicación de métodos probabilísticos", Tesis de grado, Departamento de Ingeniería Mecánica de Fluidos, Universidad Nacional Mayor de San Marcos, Lima, 1987.

[8] Ley de Recursos Hidricos, Ley 29338, 2009.

[9] A. Mejía, "Balance hídrico de la cuenca Chumbao, Andahuaylas", Administración técnica distrito de riego, Apurímac, Informe técnico, 1, 1999.

[10] R. Pinedo, "Zonificación como base para el ordenamiento territorial del municipio Valle de Ángeles, Honduras", Tesis de Posgrado, Departamento de Educación para el Desarrollo y la Conservación, CATIE, Turrialba, 2006.

[11] Reglamento de la Ley de Recursos Hídricos, Ley N²9338, 2010. 